\title{
ANNIHILATORS OF PERMUTATION MODULES
}

\author{
STEPHEN DOTY AND KATHRYN NYMAN
}

\begin{abstract}
Permutation modules are fundamental in the representation theory of symmetric groups $\mathfrak{S}_{n}$ and their corresponding Iwahori-Hecke algebras $\mathscr{H}=\mathscr{H}\left(\mathfrak{S}_{n}\right)$. We find an explicit combinatorial basis for the annihilator of a permutation module in the "integral" case - showing that it is a cell ideal in G.E. Murphy's cell structure of $\mathscr{H}$. The same result holds whenever $\mathscr{H}$ is semisimple, but may fail in the non-semisimple case.
\end{abstract}

\section{INTRODUCTION}

Let $R$ be a commutative ring with 1 and fix an invertible element $q \in R$. The representation theory of symmetric groups $\mathfrak{S}_{n}$ and the corresponding Iwahori-Hecke algebras $\mathscr{H}_{R, q}=\mathscr{H}_{R, q}\left(\mathfrak{S}_{n}\right)$ starts with the transitive permutation modules $M_{R}^{\lambda}$ indexed by partitions $\lambda$. There are also twisted versions $\widetilde{M}_{R}^{\lambda}$ and the theory may equivalently be approached through the $\widetilde{M}_{R}^{\lambda}$ instead of the $M_{R}^{\lambda}$. The purpose of this paper is to give explicit combinatorial bases for the annihilators of $M_{R}^{\lambda}$ and $\widetilde{M}_{R}^{\lambda}$ in two cases:

(i) the coefficient ring is the ring $R=\mathbb{Z}\left[v, v^{-1}\right]$ of "Laurent polynomials," where $v$ is an indeterminate and $q=v^{2}$;

(ii) the coefficient ring is a field $R$ such that $\mathscr{H}_{R, q}$ is semisimple.

The result is essentially the same in both cases; (i) is obtained in Theorem 7.3 by a refinement of an argument of Härterich [8], and (ii) is obtained in Theorem 5.2. It turns out that in these cases the annihilator of $M_{R}^{\lambda}$ is a cell ideal with respect to Murphy's cellular basis of $\mathscr{H}_{R, q}$. The result does not necessarily hold in case $\mathscr{H}_{R, q}$ is not semisimple; see Example 7.4.

In case $v=1$ the algebra $\mathscr{H}_{R, q}$ is isomorphic to the group algebra $R \mathfrak{S}_{n}$ of the symmetric group $\mathfrak{S}_{n}$; so by specializing $v$ to 1 we obtain

Date: 21 April 2009.

2000 Mathematics Subject Classification. Primary $20 \mathrm{~B} 30$.

Key words and phrases. Hecke algebra, symmetric group, permutation module, Murphy basis, cellular algebra. 
corresponding results on annihilators of permutation modules for symmetric groups. Specifically, we obtain a description of $\operatorname{Ann}_{R \mathfrak{S}_{n}} M_{R}^{\lambda}$ in case (i) $R=\mathbb{Z}$, or (ii) $R$ is a field such that $R \mathfrak{S}_{n}$ is semisimple.

Theorem 5.2 is easily derived from a lemma of [8] along with properties of cellular bases. To prove Theorem 7.3 we exploit Schur-Weyl duality between $\mathscr{H}$ and the quantized enveloping algebra $\mathbf{U}\left(\mathfrak{g l}_{n}\right)$. This gives an "integral" embedding between certain permutation modules which seems to be new; see Lemma 6.4 for the precise statement. This result, which may be of some interest in its own right, is the key step in the proof of Theorem $[7.3$.

\section{Symmetric groups and tableaux}

We denote by $\mathfrak{S}_{S}$ the symmetric group consisting of all bijections of a given set $S$; in particular we set $\mathfrak{S}_{n}=\mathfrak{S}_{\{1, \ldots, n\}}$. We adopt the convention that elements of $\mathfrak{S}_{n}$ act on the right of their arguments, so that compositions of permutations are read from left to right. In other words, if $\sigma, \tau \in \mathfrak{S}_{n}$, then $i(\sigma \tau)=(i \sigma) \tau$, for any $i \in\{1, \ldots, n\}$.

We write $\lambda \vDash n$ to indicate that $\lambda$ is a composition of $n$, meaning that $\lambda$ is an infinite sequence $\left(\lambda_{1}, \lambda_{2}, \ldots\right)$ of nonnegative integers such that $\sum \lambda_{i}=n$. The individual $\lambda_{i}$ are the parts of $\lambda$, and the largest index $\ell$ such that $\lambda_{j}=0$ for all $j>\ell$ is the length, or number of parts, of $\lambda$. Zeros at the end of $\lambda$ are usually omitted. Any composition $\lambda$ may be sorted into a unique partition $\lambda^{+}$, in which the parts are in non-strict descending order. We write $\lambda \vdash n$ to indicate that $\lambda$ is a partition of $n$. When $\lambda \vdash n$, we denote by $\lambda^{\prime}$ the transposed partition, whose Young diagram is obtained from the Young diagram of $\lambda$ by writing its rows as columns.

Recall that compositions are partially ordered by dominance: given $\lambda, \mu \vDash n$, one writes $\lambda \unrhd \mu(\lambda$ dominates $\mu)$ if $\sum_{i \leqslant j} \lambda_{i} \geqslant \sum_{i \leqslant j} \mu_{i}$ for all $j$, and one writes $\lambda \triangleright \mu(\lambda$ strictly dominates $\mu)$ if $\lambda \unrhd \mu$ and the inequality $\sum_{i \leqslant j} \lambda_{i} \geqslant \sum_{i \leqslant j} \mu_{i}$ is strict for at least one $j$. The notations $\mu \unlhd \lambda, \mu \triangleleft \lambda$ are respectively equivalent to $\lambda \unrhd \mu, \lambda \triangleright \mu$. Furthermore, we note that the dominance relation reverses when taking transposes:

$$
\lambda^{\prime} \unlhd \mu^{\prime} \quad \text { if and only if } \lambda \unrhd \mu
$$

for $\lambda, \mu \vdash n$.

Let $\lambda \vDash n$. A $\lambda$-tableau $t$ is a numbering of the boxes in the Young diagram of $\lambda$ by the numbers $1, \ldots, n$ such that each number appears just once. One says that $t$ is row-standard if the numbers in each row are increasing read from left to right, and standard if $t$ is row-standard and the numbers in each column are increasing read from top to bottom. 
The group $\mathfrak{S}_{n}$ acts naturally on the set of tableaux, on the right, as permutations of the numbering.

\section{Murphy's BASEs of the Hecke Algebra}

Recall the definition of $\mathfrak{S}_{n}$ as a Coxeter group: it is the group given by the generators $s_{1}, \ldots, s_{n-1}$ subject to the relations

(S1) $\quad s_{i}^{2}=1$;

(S2) $\quad s_{i} s_{j} s_{i}=s_{j} s_{i} s_{j} \quad$ if $|i-j|=1$;

(S3) $\quad s_{i} s_{j}=s_{j} s_{i} \quad$ if $|i-j|>1$.

We may (and will) identify the generator $s_{i}$ with the transposition interchanging $i, i+1$ and fixing all other elements of $\{1, \ldots, n\}$. Every element of $\mathfrak{S}_{n}$ is expressible (in many ways) as a product of the form $w=s_{i_{1}} \cdots s_{i_{k}}$. Let $\ell(w)$, the length of $w$, be the minimum value of $k$ in all such expressions. Any expression of the form $w=s_{i_{1}} \cdots s_{i_{k}}$ in which $k=\ell(w)$ is reduced.

Let $R$ be any commutative ring with 1 , and fix an invertible element $q$ of $R$. The Iwahori-Hecke algebra, $\mathscr{H}_{R, q}=\mathscr{H}_{R, q}\left(\mathfrak{S}_{n}\right)$ is the associative $R$-algebra with 1 given by generators $T_{1}, T_{2}, \ldots, T_{n-1}$ satisfying the relations

(H2) $\quad T_{i} T_{j} T_{i}=T_{j} T_{i} T_{j} \quad$ if $|i-j|=1$;

(H3) $\quad T_{i} T_{j}=T_{j} T_{i} \quad$ if $|i-j|>1$.

In order to simplify notation, we shall henceforth write $\mathscr{H}_{R}$ instead of $\mathscr{H}_{R, q}$, letting $q$ be understood.

Remark 3.1. Some authors use a different, but equivalent, quadratic relation in place of $(\mathrm{H} 1):\left(T_{i}-v\right)\left(T_{i}+v^{-1}\right)=0$. Setting $v=q^{\frac{1}{2}}$ and extending the scalars to include $q^{\frac{1}{2}}$, one can see that this version is isomorphic to the one defined above. The results of this paper hold for the alternative version of $\mathscr{H}_{R}$, although many of the specific formulas are somewhat different.

If $w=s_{i_{1}} \cdots s_{i_{k}}$ is a reduced expression for $w \in \mathfrak{S}_{n}$, one defines

$$
T_{w}=T_{i_{1}} \cdots T_{i_{k}} .
$$

In particular, $T_{i d}=1$ and $T_{s_{i}}=T_{i}$. The element $T_{w}$ is well defined independently of the choice of reduced expression for $w$ and satisfies

$$
T_{w} T_{s_{i}}= \begin{cases}T_{w s_{i}} & \text { if } \ell\left(w s_{i}\right)>\ell(w) \\ q T_{w s_{i}}+(q-1) T_{w} & \text { if } \ell\left(w s_{i}\right)<\ell(w) .\end{cases}
$$


The elements $T_{w}$, for $w \in \mathfrak{S}_{n}$, form an $R$-basis of $\mathscr{H}_{R}$; in particular $\mathscr{H}_{R}$ is free as an $R$-module. The generator $T_{i}=T_{s_{i}}$ is invertible in $\mathscr{H}_{R}$, with

$$
T_{i}^{-1}=q^{-1}\left(T_{i}-q+1\right)
$$

for all $i=1, \ldots, n-1$. It follows that any $T_{w}$ is invertible, with

$$
T_{w}^{-1}=T_{i_{k}}^{-1} \cdots T_{i_{1}}^{-1}
$$

where $w=s_{i_{1}} \cdots s_{i_{k}}$ is a reduced expression for $w$.

We refer the reader to [13, Lemma 2.3] for a proof of the following well known result.

Lemma 3.2. Suppose $R$ is an integral domain. Let $*, \dagger, \sharp$ be the maps $\mathscr{H}_{R} \rightarrow \mathscr{H}_{R}$ defined on basis elements by the rules

$$
\begin{aligned}
& *: T_{w} \rightarrow T_{w^{-1}} ; \\
& \dagger: T_{w} \rightarrow(-q)^{\ell(w)} T_{w}^{-1} ; \\
& \sharp: T_{w} \rightarrow(-q)^{\ell(w)}\left(T_{w^{-1}}\right)^{-1}
\end{aligned}
$$

for $w \in \mathfrak{S}_{n}$, extended to $\mathscr{H}_{R}$ by linearity. Then $*$ and $\dagger$ are commuting anti-involutions of $R$-algebras. The map $\sharp$ is the composite of $*$ and $\dagger$; thus $\sharp$ is an involution of $R$-algebras.

We denote the image of any $h \in \mathscr{H}_{R}$ under the maps $*, \dagger$, and $\sharp$ by $h^{*}, h^{\dagger}$, and $h^{\sharp}$ respectively.

We now recall some results of Murphy [12, 13], which provide two cellular bases of $\mathscr{H}_{R}$. Let $\lambda \vDash n$, and let $t^{\lambda}$ be the tableau of shape $\lambda$ in which the numbers $1, \ldots, n$ have been inserted in the boxes in order from left to right along the rows. Let $\mathfrak{S}_{\lambda}$ be the row stabilizer of $t^{\lambda}$. Then

$$
\mathfrak{S}_{\lambda}=\mathfrak{S}_{\left\{1, \ldots, \lambda_{1}\right\}} \times \mathfrak{S}_{\left\{\lambda_{1}+1, \ldots, \lambda_{1}+\lambda_{2}\right\}} \times \cdots
$$

is a Young subgroup of $\mathfrak{S}_{n}$. Define elements

$$
x_{\lambda}=\sum_{w \in \mathfrak{S}_{\lambda}} T_{w} ; \quad y_{\lambda}=\sum_{w \in \mathfrak{S}_{\lambda}}(-q)^{-\ell(w)} T_{w} .
$$

Given a tableau $t$ of shape $\lambda$, for $\lambda \vDash n$, let $d(t)$ be the unique element of $\mathfrak{S}_{n}$ such that $t=t^{\lambda} d(t)$. Given any pair $s, t$ of row-standard $\lambda$ tableaux, following Murphy we set

$$
x_{s t}=T_{d(s)}^{*} x_{\lambda} T_{d(t)}, \quad y_{s t}=T_{d(s)}^{*} y_{\lambda} T_{d(t)} .
$$

Since $x_{\lambda}$ and $y_{\lambda}$ are invariant under $*$, it follows that

$$
x_{s t}^{*}=x_{t s} ; \quad y_{s t}^{*}=y_{t s}
$$


for any pair $s, t$ of row-standard $\lambda$-tableaux. For any $\lambda \vdash n$ let $\operatorname{Tab}(\lambda)$ be the set of standard $\lambda$-tableaux, and set

$$
\mathscr{H}_{R}[\triangleright \lambda]=\sum_{a, b \in \operatorname{Tab}(\mu), \mu \triangleright \lambda} R x_{a b} ; \quad \mathscr{H}_{R}[\unrhd \lambda]=\sum_{a, b \in \operatorname{Tab}(\mu), \mu \unrhd \lambda} R x_{a b} .
$$

It follows from the first equality in (8) and part (b) of the next result that $\mathscr{H}_{R}[\triangleright \lambda]$ and $\mathscr{H}_{R}[\unrhd \lambda]$ are both two-sided ideals of $\mathscr{H}_{R}$.

Theorem 3.3. [13, Theorem 4.17 and Theorem 5.1] Assume that $R$ is an integral domain.

(a) The set $\left\{x_{s t}: s, t \in \operatorname{Tab}(\lambda), \lambda \vdash n\right\}$ is an $R$-basis of $\mathscr{H}_{R}$.

(b) If $\lambda \vdash n$, for any $h \in \mathscr{H}_{R}, s, t \in \operatorname{Tab}(\lambda)$ we have

$$
x_{s t} h=\sum_{u \in \operatorname{Tab}(\lambda)} r_{h}(t, u) x_{s u} \quad\left(\bmod \mathscr{H}_{R}[\triangleright \lambda]\right)
$$

where $r_{h}(t, u) \in R$ is independent of $s$.

Note that by applying $*$ the equality in the theorem may be written in the equivalent form

$$
h^{*} x_{t s}=\sum_{u \in \operatorname{Tab}(\lambda)} r_{h}(t, u) x_{u s} \quad\left(\bmod \mathscr{H}_{R}[\triangleright \lambda]\right) .
$$

This is used, for instance, in proving that $\mathscr{H}_{R}[\triangleright \lambda]$ and $\mathscr{H}_{R}[\unrhd \lambda]$ are two-sided ideals of $\mathscr{H}_{R}$.

\section{Remark 3.4.}

(a) The basis in part (a) of the theorem is a cellular basis in the sense of [6]. This follows from the first equality in (8) and part (b) of the theorem.

(b) For the sake of completeness, we mention that the theorem remains true if one replaces $x_{s t}$ by $y_{s t}$ throughout. Thus

$$
\left\{y_{s t}: s, t \in \operatorname{Tab}(\lambda), \lambda \vdash n\right\}
$$

is another cellular basis of $\mathscr{H}_{R}$. We will not need this fact in the paper.

(c) By applying the involution $\sharp$ to any cellular basis of $\mathscr{H}_{R}$, we obtain another cellular basis. Thus

$$
\left\{x_{s t}^{\sharp}: s, t \in \operatorname{Tab}(\lambda), \lambda \vdash n\right\}, \quad\left\{y_{s t}^{\sharp}: s, t \in \operatorname{Tab}(\lambda), \lambda \vdash n\right\}
$$

are both cellular bases of $\mathscr{H}_{R}$.

For $\lambda \vdash n$, following [6] we let $C_{R}(\lambda)$ be the free $R$-module with basis $\left\{c_{t}: t \in \operatorname{Tab}(\lambda)\right\}$. We define an action of $\mathscr{H}_{R}$ on $C_{R}(\lambda)$ by the rule

$$
c_{t} h=\sum_{u \in \operatorname{Tab}(\lambda)} r_{h}(t, u) c_{u}
$$


for any $t \in \operatorname{Tab}(\lambda)$. The element $r_{h}(t, u) \in R$ is determined as in part (b) of Theorem 3.3. The $\mathscr{H}_{R}$-modules $C_{R}(\lambda)$ are known as cell modules in the terminology of [6]. Note that for any given fixed $s \in$ $\operatorname{Tab}(\lambda)$, the right $\mathscr{H}_{R}$-module $C_{R}(\lambda)$ is isomorphic with the submodule of $\mathscr{H}_{R}[\unrhd \lambda] / \mathscr{H}_{R}[\triangleright \lambda]$ spanned by the set of all right cosets of the form

$$
x_{s t}+\mathscr{H}_{R}[\triangleright \lambda] \quad(t \in \operatorname{Tab}(\lambda)) .
$$

Murphy has identified the cell modules for $\mathscr{H}_{R}$ with respect to the cellular basis of Theorem 3.3. In [13, Theorem 5.3] he proves:

$$
C_{R}(\lambda) \simeq S_{\lambda, R} \text { for any } \lambda \vdash n .
$$

Here $S_{\lambda, R}$ is the linear dual $\operatorname{Hom}_{R}\left(S_{R}^{\lambda}, R\right)$ of the Specht module $S_{R}^{\lambda}$.

The module $S_{R}^{\lambda}$, which is a $q$-analogue of the corresponding Specht module for the symmetric group $\mathfrak{S}_{n}$, was introduced in [1, §4]. Although we need only generic properties of Specht modules associated to their interpretation as cell modules, some readers may prefer an explicit construction. One approach is to define $S_{R}^{\lambda}$ as the following right ideal of $\mathscr{H}_{R}$ :

$$
S_{R}^{\lambda}:=x_{\lambda} T_{w_{\lambda}} y_{\lambda^{\prime}} \mathscr{H}_{R},
$$

where the element $w_{\lambda}$ is the unique element of $\mathfrak{S}_{n}$ such that $t^{\lambda} w_{\lambda}=t_{\lambda}$. Here $t_{\lambda}$ is the tableau of shape $\lambda$ in which the numbers $1, \ldots, n$ have been inserted in the boxes in order from top to bottom in the columns.

\section{Permutation modules}

In [1], 2], Dipper and James studied the right ideals $M_{R}^{\lambda}:=x_{\lambda} \mathscr{H}_{R}$, for $\lambda \vDash n$, noting that they are $q$-analogues of the classical permutation modules (see [9]) for symmetric groups. They also studied the right ideals $\widetilde{M}_{R}^{\lambda}:=y_{\lambda} \mathscr{H}_{R}$; these are $q$-analogues of the signed permutation modules for symmetric groups. We wish to study the annihilators of these $\mathscr{H}_{R}$-modules.

By [2, (2.1)], there exist elements $r_{\lambda}, r_{\lambda}^{\prime} \in R$ such that $r_{\lambda} r_{\lambda}^{\prime}=1$ and

$$
x_{\lambda}^{\sharp}=r_{\lambda} y_{\lambda} ; \quad y_{\lambda}^{\sharp}=r_{\lambda}^{\prime} x_{\lambda} .
$$

Given any right $\mathscr{H}_{R^{-}}$module $N$, we obtain a new right $\mathscr{H}_{R^{-}}$module $N^{\sharp}$ in the usual way, by letting $N^{\sharp}=N$ and twisting the original $\mathscr{H}_{R}$-action by the automorphism $\sharp$. On the other hand, if $N$ is a right ideal of $\mathscr{H}_{R}$ then $N^{\sharp}=\left\{n^{\sharp}: n \in N\right\}$ is another right ideal of $\mathscr{H}_{R}$. Thus, in the case when $N$ is a right ideal, the notation $N^{\sharp}$ has two meanings. However, the reader can easily check that the two possible interpretations lead to 
isomorphic right $\mathscr{H}_{R^{-}}$modules. From (12), it follows immediately that as right $\mathscr{H}_{R}$-modules we have an isomorphism

$$
\left(M_{R}^{\lambda}\right)^{\sharp} \simeq \widetilde{M}_{R}^{\lambda}
$$

for any $\lambda \vDash n$. Thus, any description of $\operatorname{Ann}_{\mathscr{H}_{R}} M_{R}^{\lambda}$ will give immediately a description of $\operatorname{Ann}_{\mathscr{H}_{R}} \widetilde{M}_{R}^{\lambda}$, simply by applying the involution $\sharp$. So we focus on obtaining a description of the former.

Let $\mathscr{H}_{R}(\lambda)$ be the subalgebra of $\mathscr{H}_{R}$ generated by all the $T_{i}$ except $T_{\lambda_{1}}, T_{\lambda_{1}+\lambda_{2}}, \ldots, T_{\lambda_{1}+\cdots+\lambda_{k}}$ where $\lambda=\left(\lambda_{1}, \lambda_{2}, \ldots, \lambda_{k}\right)$ has length $k$. By [11, Corollary 1.14], there are two one-dimensional representations $1_{\mathscr{H}_{R}}$ and $\varepsilon_{\mathscr{H}_{R}}$ of $\mathscr{H}_{R}$ defined on basis elements by

$$
1_{\mathscr{H}_{R}}\left(T_{w}\right)=q^{\ell(w)} ; \quad \varepsilon_{\mathscr{H}_{R}}\left(T_{w}\right)=(-1)^{\ell(w)}
$$

for any $w \in \mathfrak{S}_{n}$. These are known as the trivial and sign representations, respectively; by abuse of notation we denote the corresponding right $\mathscr{H}_{R}$-modules by the same symbols. As right $\mathscr{H}_{R}$-modules, we have isomorphisms

$$
M_{R}^{\lambda} \simeq 1_{\mathscr{H}_{R}} \otimes_{\mathscr{H}_{R}(\lambda)} \mathscr{H}_{R}, \quad \widetilde{M}_{R}^{\lambda} \simeq \varepsilon_{\mathscr{H}_{R}} \otimes_{\mathscr{H}_{R}(\lambda)} \mathscr{H}_{R}
$$

thus justifying the terminology "permutation" and "signed permutation" for these modules.

Let $\lambda \vDash n$. For typographical reasons, we denote by $x_{\lambda t}$ the element $x_{s t}$ with $s=t^{\lambda}$. Thus $x_{\lambda t}=x_{\lambda} T_{d(t)}$, for any row-standard tableau $t$ of shape $\lambda$. We will need the following result of Dipper and James, which gives a basis of $M_{R}^{\lambda}$ and determines the action of $\mathscr{H}_{R}$ on basis elements.

Lemma 4.1. [1, Lemma 3.2] Assume that $R$ is an integral domain, and let $\lambda \vDash n$.

(a) $\left\{x_{\lambda t}: t\right.$ row-standard of shape $\left.\lambda\right\}$ is an $R$-basis of $M_{R}^{\lambda}=x_{\lambda} \mathscr{H}_{R}$.

(b) Suppose $t$ is row-standard of shape $\lambda$, and set $u=t s_{i}$. Let $\operatorname{row}_{t}(j)$ be the row index of $j$ in $t$. Then

$$
x_{\lambda t} T_{i}= \begin{cases}q x_{\lambda t} & \text { if } \operatorname{row}_{t}(i)=\operatorname{row}_{t}(i+1) \\ x_{\lambda u} & \text { if } \operatorname{row}_{t}(i)<\operatorname{row}_{t}(i+1) \\ q x_{\lambda u}+(q-1) x_{\lambda t} & \text { if } \operatorname{row}_{t}(i)>\operatorname{row}_{t}(i+1) .\end{cases}
$$

Note that for $\lambda \vDash n, M_{R}^{\lambda} \simeq M_{R}^{\lambda^{+}}$, where $\lambda^{+}$is the unique partition in the $\mathfrak{S}_{n^{-}}$orbit of $\lambda$. Thus, when considering the annihilator of $M_{R}^{\lambda}$, it is enough to restrict our attention to the case in which $\lambda \vdash n$. 


\section{The AnNihilator In THE SEMisimple CASE}

We will require a preparatory lemma of Härterich. To formulate it, we need to extend the dominance order on compositions to the set of row-standard tableaux, as follows. Let $t$ be a row-standard $\lambda$-tableau, where $\lambda \vDash n$. For any $j \leqslant n$ denote by $t_{\downarrow j}$ the row-standard tableau that results from throwing away all boxes of $t$ containing a number bigger than $j$. Let $\left[t_{\downarrow j}\right]$ be the corresponding composition of $j$ (the composition defining the shape of $t_{\downarrow j}$ ). Given row-standard tableaux $s$ and $t$ with the same number $n$ of boxes, define

$s \unrhd t$ if for each $j \leqslant r,\left[s_{\downarrow j}\right] \unrhd\left[t_{\downarrow j}\right]$;

$s \triangleright t$ if for each $j \leqslant r,\left[s_{\downarrow j}\right] \triangleright\left[t_{\downarrow j}\right]$.

Note that if $s$ and $t$ are standard tableaux, respectively of shape $\lambda$ and $\mu$, where $\lambda$ and $\mu$ are partitions of $n$, then $s \unrhd t$ if and only if $t^{\prime} \unrhd s^{\prime}$. Here $t^{\prime}$ denotes the transposed tableau of $t$, obtained from $t$ by writing its rows as columns. The dominance order on tableaux extends naturally to pairs of tableaux, by defining:

$$
(s, t) \unrhd(u, w) \text { iff } s \unrhd u \text { and } t \unrhd w .
$$

For a set $P$ of partitions of $n$, set $\mathscr{H}_{R}[P]=\sum_{s, t} R x_{s t}$, where the sum is taken over the set of pairs $s, t \in \operatorname{Tab}(\mu)$, for $\mu \in P$. If $P$ is closed with respect to $\unrhd$ (i.e., $\nu \vdash n, \mu \in P$, and $\nu \unrhd \mu$ implies $\nu \in P$ ), then $\mathscr{H}_{R}[P]$ is a two sided ideal of $\mathscr{H}_{R}$. For $\lambda \vdash n$, the set $P=\left\{\mu \vdash n: \mu \nsupseteq \lambda^{\prime}\right\}$ is closed with respect to $\unrhd$, so $\mathscr{H}_{R}\left[\not \lambda^{\prime}\right]$ is a two-sided ideal of $\mathscr{H}_{R}$. Thus

$$
\mathscr{H}_{R}\left[\not \lambda^{\prime}\right]^{\sharp}=\left\{h^{\sharp} \mid h \in \mathscr{H}_{R}\left[\not \lambda^{\prime}\right]\right\}
$$

is also a two-sided ideal of $\mathscr{H}_{R}$. We remark that these ideals are free as $R$-modules and have $R$-rank $\sum_{\mu^{\prime} \not \lambda^{\prime}}\left(\operatorname{dim}_{R} S_{R}^{\mu}\right)^{2}$. This follows from the cellular axioms and the various identifications made above.

The following result is a variant of [8, Lemma 3].

Lemma 5.1 (Härterich). Let $R$ be an integral domain. For any $\lambda \vdash n$ we have $\mathscr{H}_{R}\left[\not \lambda^{\prime}\right]^{\sharp} \subseteq \operatorname{Ann}_{\mathscr{H}_{R}} M_{R}^{\lambda}$.

Proof. Let $a, b \in \operatorname{Tab}(\mu)$ where $\mu \nsupseteq \lambda^{\prime}$, and let $c$ be any row-standard $\lambda$-tableau. We have $c \nsupseteq a^{\prime}$, since otherwise $\lambda=\left[c_{\downarrow n}\right] \unlhd\left[a_{\downarrow n}^{\prime}\right]=\mu^{\prime}$. By Lemma 4.12 of [13], $x_{a b}^{\dagger} x_{c \lambda}=0$. Applying $*$, we have $x_{c \lambda}^{*}\left(x_{a b}^{\dagger}\right)^{*}=$ $x_{\lambda c} x_{a b}^{\sharp}=0$. Now, since $\left\{x_{\lambda c}: c\right.$ is a row-standard $\lambda$-tableau $\}$ is a basis of $M_{R}^{\lambda}$, we have $x_{a b}^{\sharp} \in \operatorname{Ann}_{\mathscr{H}_{R}} M_{R}^{\lambda}$ for $a, b$ of shape $\mu \nsupseteq \lambda^{\prime}$.

Theorem 5.2. Let $R$ be a field, and suppose that $\mathscr{H}_{R}$ is semisimple. Then, for any $\lambda \vdash n$, equality holds in the inclusion in the preceding lemma; i.e., we have $\mathscr{H}_{R}\left[\not \lambda^{\prime}\right]^{\sharp}=\operatorname{Ann}_{\mathscr{H}_{R}} M_{R}^{\lambda}$. 
Proof. The semisimplicity of $\mathscr{H}_{R}$ implies that $M_{R}^{\lambda}$ is completely reducible as an $\mathscr{H}_{R}$-module, with irreducible factors of the form $S_{R}^{\mu}$ for various $\mu \unrhd \lambda$. By the $q$-analogue of Young's rule [13, Theorem 7.2], each $S_{R}^{\mu}$ for any $\mu$ satisfying $\mu \unrhd \lambda$ occurs at least once in $M_{R}^{\lambda}$. Thus, it follows by the theory of semisimple algebras that

$$
\operatorname{dim}_{R}\left(\mathscr{H}_{R} / \operatorname{Ann}_{\mathscr{H}_{R}} M_{R}^{\lambda}\right) \geqslant \sum_{\mu \unrhd \lambda}\left(\operatorname{dim}_{R} S_{R}^{\mu}\right)^{2},
$$

or, equivalently,

$$
\operatorname{dim}_{R} \operatorname{Ann}_{\mathscr{H}_{R}} M_{R}^{\lambda} \leqslant \sum_{\mu \nsubseteq \lambda}\left(\operatorname{dim}_{R} S_{R}^{\mu}\right)^{2} .
$$

But the remark at the end of the paragraph preceding Lemma 5.1 gives the equality

$$
\sum_{\mu^{\prime} \pitchfork \lambda^{\prime}}\left(\operatorname{dim}_{R} S_{R}^{\mu}\right)^{2}=\operatorname{dim}_{R} \mathscr{H}_{R}\left[\not \nexists \lambda^{\prime}\right]^{\sharp}
$$

and applying the equivalence between the two conditions $\mu^{\prime} \unlhd^{\prime}$ and $\mu \nsupseteq \lambda$, we conclude that

$$
\operatorname{dim}_{R} \operatorname{Ann}_{\mathscr{H}_{R}} M_{R}^{\lambda} \leqslant \operatorname{dim}_{R} \mathscr{H}_{R}\left[\not \nexists \lambda^{\prime}\right]^{\sharp} .
$$

Finally, Lemma 5.1 gives the opposite inequality, thus proving the result.

Remark 5.3. Let $R$ be a field. It is well known [4, Theorem 4.3] that $\mathscr{H}_{R}$ is semisimple unless one of the following holds:

(1) $q \neq 1$ and $q$ is a primitive $e$ th root of 1 , where $e \leqslant n$;

(2) $q=1$ and the characteristic of $R$ is $\leqslant n$.

In case $\mathscr{H}_{R}$ is not semisimple, the inclusion of Lemma 5.1 may be strict (see Example 7.4).

\section{TENSOR SPACE}

Henceforth we will work over the $\operatorname{ring} \mathcal{A}=\mathbb{Z}\left[v, v^{-1}\right]$ where $v$ is an indeterminate, with quotient field $\mathbb{Q}(v)$. We set $q=v^{2}$ and consider the Hecke algebra $\mathscr{H}_{\mathcal{A}}$ over the ring $\mathcal{A}$, defined by the generators and relations as in $\S 3$. For any commutative ring $R$ with a chosen invertible element $v$ we set $q=v^{2}$ in $R$. Then

$$
R \otimes_{\mathcal{A}}\left(\mathscr{H}_{\mathcal{A}}\right) \simeq \mathscr{H}_{R}
$$

where $R$ is regarded as an $\mathcal{A}$-algebra by means of the ring homomorphism $\mathcal{A} \rightarrow R$ sending $v \in \mathcal{A}$ to $v \in R$. The isomorphism in (17) is determined by sending $1 \otimes T_{i} \rightarrow T_{i}$ for all $i=1, \ldots, n-1$. Moreover, we have

$$
R \otimes_{\mathcal{A}}\left(M_{\mathcal{A}}^{\lambda}\right) \simeq M_{R}^{\lambda}
$$


for any $\lambda \vDash n$, and any $R$. Our goal is to determine the annihilator of the integral permutation module $M_{\mathcal{A}}^{\lambda}$.

We will need the Drinfeld-Jimbo quantized enveloping algebra corresponding to the Lie algebra $\mathfrak{g l}_{m}$ for $m \geqslant 2$. Let $Y$ be the free abelian group with basis $H_{1}, \ldots, H_{m}$. Let $\varepsilon_{1}, \ldots, \varepsilon_{m} \in X:=Y^{*}$ be the corresponding dual basis; $\varepsilon_{i}$ is given by $\varepsilon_{i}\left(H_{j}\right):=\delta_{i, j}$ for $j=1, \ldots, m$. For $i=1, \ldots, m-1$ let $\alpha_{i} \in X$ be given by $\alpha_{i}:=\varepsilon_{i}-\varepsilon_{i+1}$. Define a partial order $\leqslant$ on $X$ by $\lambda \leqslant \mu$ if and only if $\mu-\lambda \in \sum_{i} \mathbb{N} \alpha_{i}$.

Define an associative algebra $\mathbf{U}=\mathbf{U}\left(\mathfrak{g l}_{n}\right)$ (with 1 ) over $\mathbb{Q}(v)$ by the generators

$$
E_{i}, F_{i} \quad(i=1, \ldots, m-1), \quad v^{h} \quad(h \in Y)
$$

subject to the defining relations

(U1) $\quad v^{0}=1, \quad v^{h} v^{h^{\prime}}=v^{h+h^{\prime}}$

(U2) $\quad v^{h} E_{i} v^{-h}=v^{\alpha_{i}(h)} E_{i}, \quad v^{h} F_{i} v^{-h}=v^{-\alpha_{i}(h)} F_{i}$

(U3) $E_{i} F_{j}-F_{j} E_{i}=\delta_{i j} \frac{K_{i}-K_{i}^{-1}}{v-v^{-1}} \quad$ where $K_{i}:=v^{H_{i}-H_{i+1}}$

(U4) $\quad E_{i}^{2} E_{j}-\left(v+v^{-1}\right) E_{i} E_{j} E_{i}+E_{j} E_{i}^{2}=0 \quad$ if $|i-j|=1$

(U5) $\quad F_{i}^{2} F_{j}-\left(v+v^{-1}\right) F_{i} F_{j} F_{i}+F_{j} F_{i}^{2}=0 \quad$ if $|i-j|=1$

(U6) $\quad E_{i} E_{j}=E_{j} E_{i}, \quad F_{i} F_{j}=F_{j} F_{i} \quad$ if $|i-j|>1$

for $1 \leqslant i, j \leqslant m-1$ and $h, h^{\prime} \in Y$. The subalgebra of $\mathbf{U}$ generated by all the $E_{i}, F_{i}, K_{i}$ (for $i=1, \ldots, m-1$ ) is denoted by $\mathbf{U}\left(\mathfrak{s l}_{m}\right)$; this is the quantized enveloping algebra corresponding to the Lie algebra $\mathfrak{s l}_{m}$.

In the remainder of this section all tensor products will be over $\mathbb{Q}(v)$ unless specified otherwise. There exist unique algebra maps $\Delta: \mathbf{U} \rightarrow$ $\mathbf{U} \otimes \mathbf{U}$ (where $\mathbf{U} \otimes \mathbf{U}$ is regarded as an algebra in the usual way) and $\epsilon: \mathbf{U} \rightarrow \mathbb{Q}(v)$ such that

$$
\begin{gathered}
\Delta\left(v^{h}\right)=v^{h} \otimes v^{h}, \quad \epsilon\left(v^{h}\right)=1 \\
\Delta\left(E_{i}\right)=E_{i} \otimes K_{i}+1 \otimes E_{i}, \quad \epsilon\left(E_{i}\right)=0 \\
\Delta\left(F_{i}\right)=F_{i} \otimes 1+K_{i}^{-1} \otimes F_{i}, \quad \epsilon\left(F_{i}\right)=0
\end{gathered}
$$

for any $i=1, \ldots, m-1$, and $h \in Y$. The map $\Delta$ defines a comultiplication and the map $\epsilon$ a counit which together define a coalgebra structure on $\mathbf{U}$. (Actually, there is a well defined antipode $S: \mathbf{U} \rightarrow \mathbf{U}^{o p p}$ which together with $\Delta, \epsilon$ give $\mathbf{U}$ a Hopf algebra structure, but we shall not need it.) The map $\Delta$ induces a map $\Delta^{(n)}: \mathbf{U} \rightarrow \mathbf{U}^{\otimes n}$ defined as the composite

$$
\mathbf{U} \stackrel{\Delta}{\longrightarrow} \mathbf{U} \otimes \mathbf{U} \stackrel{\Delta \otimes 1}{\longrightarrow} \mathbf{U} \otimes \mathbf{U} \otimes \mathbf{U} \stackrel{\Delta \otimes 1 \otimes 1}{\longrightarrow} \cdots \stackrel{\Delta \otimes 1 \otimes \cdots \otimes 1}{\longrightarrow} \mathbf{U}^{\otimes n}
$$


for $n \geqslant 2$. Note that $(\Delta \otimes 1) \Delta=(1 \otimes \Delta) \Delta$, etc. We have $\Delta=\Delta^{(2)}$ and

$$
\begin{gathered}
\Delta^{(n)}\left(v^{h}\right)=v^{h} \otimes v^{h} \otimes \cdots \otimes v^{h} \\
\Delta^{(n)}\left(E_{i}\right)=E_{i} \otimes K_{i} \otimes \cdots \otimes K_{i}+1 \otimes E_{i} \otimes K_{i} \otimes \cdots \otimes K_{i}+ \\
\cdots+1 \otimes \cdots \otimes 1 \otimes E_{i} \\
\Delta^{(n)}\left(F_{i}\right)=F_{i} \otimes 1 \otimes \cdots \otimes 1+K_{i}^{-1} \otimes F_{i} \otimes 1 \otimes \cdots \otimes 1+ \\
\cdots+K_{i}^{-1} \otimes \cdots \otimes K_{i}^{-1} \otimes F_{i} .
\end{gathered}
$$

The map $\Delta^{(n)}$ is used to put a $\mathbf{U}$-module structure on the $n$th tensor power of a given $\mathbf{U}$-module.

Set $V=\mathbb{Q}(v)^{m}$ with canonical basis $\left\{e_{1}, \ldots, e_{m}\right\}$. Define a left action of $\mathbf{U}$ on $V$ by

$$
E_{i} e_{j}=\delta_{j, i+1} e_{i} ; \quad F_{i} e_{j}=\delta_{j, i} e_{i+1} ; \quad v^{h} e_{j}=v^{\varepsilon_{j}(h)} e_{j}
$$

for $j=1, \ldots, m, i=1, \ldots, m-1, h \in Y$. This action makes $V$ into a $\mathrm{U}$-module, and by applying $\Delta^{(n)}$ one makes the tensor power $V^{\otimes n}$ into a U-module as well.

Let $I(m, n)$ be the set of all finite sequences $\mathbf{i}=\left(i_{1}, \ldots, i_{n}\right)$ where each $i_{j} \in\{1, \ldots, m\}$. Set

$$
e_{\mathbf{i}}:=e_{i_{1}} \otimes e_{i_{2}} \otimes \cdots \otimes e_{i_{n}}
$$

in terms of this notation $\left\{e_{\mathbf{i}}: \mathbf{i} \in I(m, n)\right\}$ is a basis for $V^{\otimes n}$. Let $\mathbf{U}^{0}$ be the subalgebra of $\mathbf{U}$ generated by the $v^{h}$ for $h \in Y$. Since

$$
v^{h} e_{\mathbf{i}}=v^{\lambda(h)} e_{\mathbf{i}}
$$

for all $h \in Y$, the vectors $e_{\mathbf{i}}$ are weight vectors (of weight $\lambda$ ) for the action of $\mathbf{U}^{0}$. Here $\lambda=\lambda_{1} \varepsilon_{1}+\cdots+\lambda_{m} \varepsilon_{m} \in X$ where each $\lambda_{k}=$ the number of $j$ such that $i_{j}=k$. It follows that

$$
V^{\otimes n}=\bigoplus_{\lambda \in X}\left(V^{\otimes n}\right)_{\lambda}
$$

where $\left(V^{\otimes n}\right)_{\lambda}$ is the $\mathbb{Q}(v)$-span of all $e_{\mathbf{i}}$ of weight $\lambda$.

The algebra $\mathbf{U}$ has $q$-analogues of the standard root vectors in $\mathfrak{g l}_{m}$, defined as follows. For a positive root $\alpha=\varepsilon_{i}-\varepsilon_{j}$ for $1 \leqslant i<j \leqslant m$, if $j-i=1$ then set $E_{i, j}=E_{i}$ and $E_{j, i}=F_{i}$. If $j-i>1$, we assume by induction that $E_{i+1, j}$ and $E_{j, i+1}$ have already been defined, and set (following Jimbo [10, Proposition 1], Xi [14, Section 5.6])

$$
E_{i, j}=v^{-1} E_{i} E_{i+1, j}-E_{i+1, j} E_{i} ; \quad E_{j, i}=v E_{j, i+1} F_{i}-F_{i} E_{j, i+1} .
$$

Set $K_{i, j}=v^{H_{i}-H_{j}}$ for each $1 \leqslant i<j \leqslant m$. We note the following results. 
Lemma 6.1. For $1 \leqslant i<j \leqslant m$ the triple $\left\{E_{i, j}, K_{i, j}, E_{j, i}\right\}$ generates a subalgebra of $\mathbf{U}$ isomorphic with $\mathbf{U}\left(\mathfrak{s l}_{2}\right)$.

Proof. Set $E=E_{i, j}, K=K_{i, j}$, and $F=E_{j, i}$ for $i<j$. We need only show that $E, K$, and $F$ satisfy the defining relations for $\mathbf{U}\left(\mathfrak{s l}_{2}\right)$ :
(a) $K E K^{-1}=v^{2} E$;
$K F K^{-1}=v^{-2} F$
(b) $E F-F E=\frac{K-K^{-1}}{v-v^{-1}}$

In the case $j-i=1$, (a) follows from (U1) and (U2) while (b) is immediate from (U3). Assume $j-i>1$, and assume that (a) and (b) hold for $E^{\prime}=E_{i+1, j}, K^{\prime}=K_{i-1, j}$, and $F^{\prime}=E_{j, i+1}$ in place of $E, K$, and $F$ respectively. Then, using 23 and the equality $K=K_{i} K^{\prime}$, we have

$$
\begin{aligned}
K E K^{-1} & =\left(K_{i} K^{\prime}\right)\left(v^{-1} E_{i} E^{\prime}-E^{\prime} E_{i}\right)\left(K_{i} K^{\prime}\right)^{-1} \\
& =v^{-1}\left(K_{i} E_{i} K_{i}^{-1}\right)\left(K^{\prime} E^{\prime} K^{\prime-1}\right)-\left(K^{\prime} E^{\prime} K^{\prime-1}\right)\left(K_{i} E_{i} K_{i}^{-1}\right) \\
& =v^{2}\left(v^{-1} E_{i} E^{\prime}-E^{\prime} E_{i}\right) \\
& =v^{2} E
\end{aligned}
$$

which proves the first relation in (a). The second relation in (a) and relation (b) are proved by similar calculations.

The following result was observed in [5, Lemma 4.2] although in a slightly different context. We adapt the argument from [5] to our situation.

Lemma 6.2. Let $\mu \in X$, and set $\mu=\sum_{k} \mu_{k} \varepsilon_{k}$. Assume $\mu_{k} \geq 0$ for $k=1, \ldots, m$. Suppose that $i<j$ and $\mu_{i}-\mu_{j}>0$. Then the action of $E_{j, i}$ gives a $\mathbb{Q}(v)$-linear injection $\left(V^{\otimes n}\right)_{\mu} \rightarrow\left(V^{\otimes n}\right)_{\mu-\varepsilon_{i}+\varepsilon_{j}}$.

Proof. It is clear that the action of $E_{j, i}$ gives a linear map from $\left(V^{\otimes n}\right)_{\mu}$ into $\left(V^{\otimes n}\right)_{\mu-\varepsilon_{i}+\varepsilon_{j}}$, so we need only to prove that the map is injective. Set

$$
E=E_{i, j}, \quad K=K_{i, j}, \quad F=E_{j, i} .
$$

By induction on $r$, one proves easily that

$$
E^{r} F-F E^{r}=\sum_{\substack{s+s^{\prime}=r-1 \\ s, s^{\prime} \geq 0}} E^{s} \frac{K-K^{-1}}{v-v^{-1}} E^{s^{\prime}}
$$

for any positive integer $r$. Fix some $0 \neq u \in\left(V^{\otimes n}\right)_{\mu}$ and choose $r$ so that $E^{r} u=0$ but $E^{s} u \neq 0$ for any $s<r$. This is possible since $E$ acts locally nilpotently on any finite-dimensional U-module. Then

$$
E^{r} F u=\left(E^{r} F-F E^{r}\right) u
$$




$$
=\sum_{\substack{s+s^{\prime}=r-1 \\ s, s^{\prime} \geq 0}} E^{s} \frac{K-K^{-1}}{v-v^{-1}} E^{s^{\prime}} u .
$$

But a calculation in $\mathbf{U}\left(\mathfrak{s l}_{2}\right)$ shows that

$$
\frac{K-K^{-1}}{v-v^{-1}} E^{s^{\prime}} u=v^{\mu_{i}-\mu_{j}+2 s^{\prime}} E^{s^{\prime}} u,
$$

and it follows that $E^{r} F u=\left(\sum_{s^{\prime}=0}^{r-1} v^{\mu_{i}-\mu_{j}+2 s^{\prime}}\right) E^{r-1} u \neq 0$. This proves that $F u=E_{j, i} u \neq 0$, so $E_{j, i}$ is injective, as desired.

Consider the action of $\mathfrak{S}_{n}$ (on the right) on $V^{\otimes n}$ by place permutation:

$$
\left(u_{1} \otimes u_{2} \otimes \cdots \otimes u_{n}\right) w=u_{1 w^{-1}} \otimes u_{2 w^{-1}} \otimes \cdots \otimes u_{n w^{-1}}
$$

for $w \in \mathfrak{S}_{n}, u_{1}, \ldots, u_{n} \in V$. Note that $\mathfrak{S}_{n}$ acts (on the right) on the set $I(m, n)$, by the rule

$$
\mathbf{i} \cdot w=\left(i_{1 w^{-1}} \cdot i_{2 w^{-1}}, \ldots, i_{n w^{-1}}\right)
$$

for $\mathbf{i}=\left(i_{1}, i_{2}, \ldots, i_{n}\right), w \in \mathfrak{S}_{n}$. With this notation, the action of $\mathfrak{S}_{n}$ on the basis elements $e_{\mathbf{i}}$ of $V^{\otimes n}$ is given by $e_{\mathbf{i}} w=e_{\mathbf{i} \cdot w}$.

We now define a right action of $\mathscr{H}=\mathscr{H}_{\mathbb{Q}(v)}$ on $V^{\otimes n}$ on basis elements by

$$
e_{\mathbf{i}} T_{k}= \begin{cases}v^{2} e_{\mathbf{i}} & \text { if } i_{k}=i_{k+1} \\ v e_{\mathbf{i} \cdot s_{k}} & \text { if } i_{k}<i_{k+1} \\ v e_{\mathbf{i} \cdot s_{k}}+\left(v^{2}-1\right) e_{\mathbf{i}} & \text { if } i_{k}>i_{k+1} .\end{cases}
$$

for any $\mathbf{i} \in I(m, n)$, and any $k=1, \ldots, n-1$. Notice that when $v=1$ this is the same as the place permutation action of $\mathfrak{S}_{n}$. One may check that the actions of $\mathscr{H}$ and $\mathbf{U}$ on $V^{\otimes n}$ commute; this goes back to [10]. The commutativity of the two actions means that a weight space $\left(V^{\otimes n}\right)_{\lambda}$ is a right $\mathscr{H}$-module, for any $\lambda \in X$.

Lemma 6.3. Let $\lambda \vDash n$, and assume $m \geqslant \ell(\lambda)$. Identify $\lambda=\left(\lambda_{1}, \ldots, \lambda_{m}\right)$ with the element $\sum_{i} \lambda_{i} \varepsilon_{i}$ of $X$. Write $M^{\lambda}:=M_{\mathbb{Q}(v)}^{\lambda}$. Then $M^{\lambda} \simeq$ $\left(V^{\otimes n}\right)_{\lambda}$ as right $\mathscr{H}$-modules.

Proof. (See [3, §2] or [7, §1].) Any $\underline{i} \in I(m, n)$ determines a unique rowstandard tableau $t(\underline{i})$, in which $j$ appears in row $k$ whenever $i_{j}=k$, for $j=1, \ldots, n$. For example,

$$
t((2,1,2,3))=\begin{array}{ll}
2 \\
\hline 1 & 3 \\
\hline 4
\end{array}
$$


Let $\lambda \vDash n$ and let $\ell(\lambda) \leq m$. Define a map $\phi:\left(V^{\otimes n}\right)_{\lambda} \rightarrow M_{R}^{\lambda}$ on a basis by

$$
\phi\left(e_{\underline{i}}\right)=v^{N} x_{\lambda t(\underline{i})},
$$

where $\underline{i} \in I(m, n)$ indexes a simple tensor $e_{i}$ of weight $\lambda$, and $N$ is the number of pairs $s<s^{\prime}$ such that $i_{s}<i_{s^{\prime}}$. The map $\phi$ gives the desired isomorphism.

The next result does not seem to have been previously observed in the literature. By specializing $v$ to 1 it gives in particular embeddings of permutation modules for $\mathbb{Q} \mathfrak{S}_{n}$.

Lemma 6.4. Suppose that $R=\mathbb{Q}(v)$ with $v$ an indeterminate, and $q=v^{2}$. If $\lambda \unrhd \mu$, then $M^{\lambda}$ is isomorphic with an $\mathscr{H}$-submodule of $M^{\mu}$.

Proof. It suffices to prove the lemma for the case $\lambda \triangleright \mu$ and $\lambda, \mu$ are adjacent in the dominance order. In this case, the Young diagram of $\lambda$ is obtained from that of $\mu$ by raising one box from the $j^{\text {th }}$ to the $i^{\text {th }}$ row, where $i<j$. This implies the statement in general, since whenever $\lambda \triangleright \mu$, one gets from $\mu$ to $\lambda$ by a finite succession of such box-raising operations.

Now, for $\lambda \triangleright \mu$ and $\lambda, \mu$ adjacent, we have $\lambda=\mu+\varepsilon_{i}-\varepsilon_{j}$, and the result follows from Lemmas 6.2 and 6.3 .

Remark 6.5. Suppose that $R$ is a field and $v=1$. It is easy to see that Lemma 6.4 may be false if the characteristic of $R$ is positive. For example, consider the map $f_{12}: M^{(3,1)} \rightarrow M^{(2,2)}$. The matrix of this map, with respect to a convenient choice of ordering of bases, is as follows:

$$
\left(\begin{array}{llllll}
1 & 1 & 0 & 1 & 0 & 0 \\
1 & 0 & 1 & 0 & 1 & 0 \\
0 & 1 & 1 & 0 & 0 & 1 \\
0 & 0 & 0 & 1 & 1 & 1
\end{array}\right)
$$

and one observes that each column has precisely two 1's, so the sum of the rows is zero in characteristic 2 . Thus, in characteristic 2 the matrix has rank strictly less than 4, and thus the map is not injective. (This example is related to Example 7.4 below.)

It is easy to construct similar examples showing that Lemma 6.4 fails in any given positive characteristic, in the $v=1$ case.

\section{ThE ANNIHILATOR IN THE INTEGRAL CASE}

In this section we work over the $\operatorname{ring} \mathcal{A}=\mathbb{Z}\left[v, v^{-1}\right]$. In Theorem 7.3 below, we describe $\operatorname{Ann}_{\mathscr{H}_{\mathcal{A}}} M_{\mathcal{A}}^{\lambda}$, using a refinement of an argument of [8]. The following result will be crucial in the proof of Theorem 7.3 ,

Lemma 7.1. If $\lambda \unrhd \mu$ for $\lambda, \mu \vdash n$, then $\operatorname{Ann}_{\mathscr{H}_{\mathcal{A}}} M_{\mathcal{A}}^{\lambda} \supseteq \operatorname{Ann}_{\mathscr{H}_{\mathcal{A}}} M_{\mathcal{A}}^{\mu}$. 
Proof. This is an immediate consequence of Lemma 6.4, since the restriction of an injective map is injective.

Next, we will need a result of Murphy. Let $a, b \in \mathscr{H}_{\mathcal{A}}$, and let $(a, b)$ denote the coefficient of $T_{1}$ in the expression $a b^{*}=\sum_{w \in \mathfrak{S}_{n}} c_{w} T_{w}$, where $c_{w} \in \mathcal{A}$. Then $($,$) is a non-degenerate, symmetric bilinear form on$ $\mathscr{H}_{\mathcal{A}}$. This bilinear form satisfies the properties

$$
(a, b d)=\left(a d^{*}, b\right) ; \quad(a, d b)=\left(d^{*} a, b\right)
$$

for any $a, b, d \in \mathscr{H}_{\mathcal{A}}$.

Lemma 7.2 (Murphy [13, Lemma 4.16]). Let $s$ and $t$ be row-standard $\mu$-tableaux and let $u, w \in \operatorname{Tab}(\lambda)$, where $\mu \vDash n$ and $\lambda \vdash n$. Then:

(a) $\left(x_{s t}, x_{u w}^{\sharp}\right)=0$ unless $\left(u^{\prime}, w^{\prime}\right) \unrhd(s, t)$;

(b) $\left(x_{u^{\prime} w^{\prime}}, x_{u w}^{\sharp}\right)= \pm v^{2 b}$ where $b=\ell\left(d\left(t_{\lambda}\right)\right)+\sum_{i \geq 1} \lambda_{i}^{\prime}\left(\lambda_{i}^{\prime}-1\right) / 2$.

The following result is an "integral" version of Theorem 5.2 ,

Theorem 7.3. For any $\lambda \vdash n$ we have $\operatorname{Ann}_{\mathscr{H}_{\mathcal{A}}} M_{\mathcal{A}}^{\lambda}=\mathscr{H}_{\mathcal{A}}\left[\not \lambda^{\prime}\right]^{\sharp}$.

Proof. By Lemma [5.1, we have already the containment $\mathscr{H}_{\mathcal{A}}\left[\AA^{\prime}\right]^{\sharp} \subseteq$ $\operatorname{Ann}_{\mathscr{H}_{\mathcal{A}}} M_{\mathcal{A}}^{\lambda}$, so we have only to prove the reverse containment. Let

$$
0 \neq a=\sum_{\substack{s, t \in \operatorname{Tab}(\lambda) \\ \lambda \vdash n}} a_{s t} x_{s t}^{\sharp} \in \operatorname{Ann}_{\mathscr{H}_{\mathcal{A}}} M_{\mathcal{A}}^{\lambda},
$$

where $a_{s t} \in \mathcal{A}$. It suffices to prove that $a_{s t}=0$ for all $s$ and $t$ of some shape $\mu \unlhd \lambda^{\prime}$.

Suppose not. By Lemma 5.1 we have $\sum_{(s, t) \in \Phi} a_{s t} x_{s t}^{\sharp} \in \operatorname{Ann}_{\mathscr{H}_{\mathcal{A}}} M_{\mathcal{A}}^{\lambda}$, hence it follows that

$$
0 \neq a_{0}=\sum_{(s, t) \in \Phi^{\mathrm{c}}} a_{s t} x_{s t}^{\sharp} \in \operatorname{Ann}_{\mathscr{H}_{\mathcal{A}}} M_{\mathcal{A}}^{\lambda},
$$

where $\Phi=\bigsqcup_{\mu \nsubseteq \lambda^{\prime}} \operatorname{Tab}(\mu) \times \operatorname{Tab}(\mu)$ and $\Phi^{\mathrm{c}}=\bigsqcup_{\mu \unlhd \lambda^{\prime}} \operatorname{Tab}(\mu) \times \operatorname{Tab}(\mu)$. Let $\left(s_{0}, t_{0}\right)$ be a minimal pair in $\Phi^{\mathrm{c}}$ such that $a_{s_{0} t_{0}} \neq 0$; i.e.,

$$
a_{s t}=0 \text { for all }(s, t) \in \Phi^{\mathrm{c}} \text { satisfying }(s, t) \triangleleft\left(s_{0}, t_{0}\right) .
$$

Let $\lambda_{0}$ be the shape of $s_{0}^{\prime}$ (= shape of $t_{0}^{\prime}$ ). Then $\lambda_{0}^{\prime}$ is the shape of $s_{0}, t_{0}$, so $\lambda_{0}^{\prime} \unlhd \lambda^{\prime}$, and hence $\lambda \unlhd \lambda_{0}$. By Lemma 7.1, anything annihilating $M_{\mathcal{A}}^{\lambda}$ also annihilates $M_{\mathcal{A}}^{\lambda_{0}}$. Thus, it follows that $x_{\lambda_{0}} T_{d\left(t_{0}^{\prime}\right)} a_{0}=0$. Hence, $T_{d\left(s_{0}^{\prime}\right)}^{*} x_{\lambda_{0}} T_{d\left(t_{0}^{\prime}\right)} a_{0}=x_{s_{0}^{\prime} t_{0}^{\prime}} a_{0}=0$. So, by the definition of the bilinear 
form, we have

$$
0=\left(x_{s_{0}^{\prime} t_{0}^{\prime}}, \sum_{(s, t) \in \Phi^{\mathrm{c}}} a_{s t} x_{s t}^{\sharp}\right)=\sum_{(s, t) \in \Phi^{\mathrm{c}}} a_{s t}\left(x_{s_{0}^{\prime} t_{0}^{\prime}}, x_{s t}^{\sharp}\right) .
$$

By Lemma $7.2(\mathrm{a})$, all the terms in the last sum are zero unless $\left(s^{\prime}, t^{\prime}\right) \unrhd$ $\left(s_{0}^{\prime}, t_{0}^{\prime}\right)$, i.e., unless $(s, t) \unlhd\left(s_{0}, t_{0}\right)$. By the minimality assumption, $a_{s t}=$ 0 for all pairs $(s, t)$ strictly less dominant than $\left(s_{0}, t_{0}\right)$. Thus, the sum $\sum_{(s, t) \in \Phi^{\mathrm{c}}} a_{s t}\left(x_{s_{0}^{\prime} t_{0}^{\prime}}, x_{s t}^{\sharp}\right)$ collapses to a single term $a_{s_{0} t_{0}}\left(x_{s_{0}^{\prime} t_{0}^{\prime}}, x_{s_{0} t_{0}}^{\sharp}\right)$, and by our assumption and Lemma 7.2(b) this term is nonzero. This is a contradiction. This contradiction establishes the desired opposite inclusion, and proves the theorem.

Example 7.4. We can now give an example to show that the annihilator of $M_{R}^{\lambda}$ depends on $R$, even when $R$ is a field. We take $v=1$, $\lambda=(2,2)$, and let $R$ be a field of characteristic 2 . It is quickly seen that the element $r=(23)+(1342)+(1243)+(14)$ (written in cycle notation) annihilates $M_{R}^{(2,2)}$ in characteristic 2. However, we claim $r$ is not in the span of the basis elements of $\mathrm{Ann}_{\mathbb{Z} \mathfrak{S}_{4}} M_{\mathbb{Z}}^{(2,2)}$ with coefficients reduced modulo 2 .

By Theorem 7.3, for standard tableaux $a=112|3| 4, b=$\begin{tabular}{|l|l|l|}
\hline$\frac{2}{4}$ & 3 \\
\hline
\end{tabular} , $c=$\begin{tabular}{|l|l|l}
\hline 1 & 2 & 4
\end{tabular} , and $d=$\begin{tabular}{|l|l|l}
\hline 1 & 3 & 4 \\
\hline 2 &
\end{tabular} , the following 10 Murphy elements form a basis for $\operatorname{Ann}_{\mathbb{Z} \mathfrak{S}_{4}} M_{\mathbb{Z}}^{(2,2)}$.

$$
\begin{aligned}
x_{a a}^{\sharp} & =\sum_{w \in \mathfrak{S}_{4}}(-1)^{\ell(w)} w \\
x_{b b}^{\sharp} & =(1)-(12)-(13)-(23)+(123)+(132) \\
x_{b c}^{\sharp} & =(34)-(12)(34)-(143)-(243)+(1243)+(1432) \\
x_{b d}^{\sharp} & =(234)-(1342)-(1423)-(24)+(13)(24)+(142) \\
x_{c c}^{\sharp} & =(1)-(12)-(14)-(24)+(124)+(142) \\
x_{c b}^{\sharp} & =(34)-(12)(34)-(134)-(234)+(1234)+(1342) \\
x_{c d}^{\sharp} & =(23)-(132)-(14)(23)-(243)+(1324)+(1432) \\
x_{d b}^{\sharp} & =(243)-(1243)-(1324)-(24)+(124)+(13)(24) \\
x_{d c}^{\sharp} & =(23)-(123)-(14)(23)-(234)+(1234)+(1423) \\
x_{d d}^{\sharp} & =(1)-(13)-(14)-(34)+(134)+(143) .
\end{aligned}
$$

It is easy to see that $r$ does not belong to the $R$-linear span of these basis elements. This shows that $\operatorname{dim}_{R} \operatorname{Ann}_{R \mathfrak{S}_{4}} M_{R}^{(2,2)} \geqslant 11$. (In fact, it equals 11.) 


\section{Applications}

We give some consequences of Theorem 7.3. As above, we work over $\mathcal{A}=\mathbb{Z}\left[v, v^{-1}\right]$ in this section. First we note the following consequence of the main results.

Remark 8.1. As already noted in Section 3, we obtain the following result immediately by applying the involution $\sharp$ to the equalities in Theorems 5.2 and 7.3 .

$$
\operatorname{Ann}_{\mathscr{H}_{R}} \widetilde{M}_{R}^{\lambda}=\mathscr{H}_{R}\left[\not \nexists \lambda^{\prime}\right]
$$

provided $\mathscr{H}_{R}$ is semisimple over a field $R$, or $R=\mathcal{A}$.

The next application is the cellularity of the algebras $\mathscr{H}_{\mathcal{A}} /\left(\operatorname{Ann}_{\mathscr{H}_{\mathcal{A}}} M_{\mathcal{A}}^{\lambda}\right)$.

Corollary 8.2. Let $\lambda \vdash n$. The quotient algebra $\mathscr{H}_{\mathcal{A}} /\left(\operatorname{Ann}_{\mathscr{H}_{\mathcal{A}}} M_{\mathcal{A}}^{\lambda}\right)$ is cellular with cell basis $\left\{x_{s t}^{\sharp}+\mathscr{H}_{\mathcal{A}}\left[\lambda^{\prime}\right]^{\sharp}: s, t \in \operatorname{Tab}(\mu), \mu \unlhd \lambda^{\prime}\right\}$.

Proof. This follows immediately from the Theorem and the theory of cellular algebras.

Finally, we observe that certain other modules have the same annihilator as $M_{\mathcal{A}}^{\lambda}$. Recall that $\mathscr{H}_{\mathcal{A}}[\unrhd \lambda]^{\sharp} / \mathscr{H}_{\mathcal{A}}[\triangleright \lambda]^{\sharp}$ is naturally a right $\mathscr{H}_{\mathcal{A}}$-module under right multiplication by elements of $\mathscr{H}_{\mathcal{A}}$ (see $\S_{3}$ ).

Corollary 8.3. For any $\lambda \vdash n$, we have the equalities

$$
\begin{aligned}
\operatorname{Ann}_{\mathscr{H}_{\mathcal{A}}} \mathscr{H}_{\mathcal{A}}[\unrhd \lambda] & =\operatorname{Ann}_{\mathscr{H}_{\mathcal{A}}}\left(\mathscr{H}_{\mathcal{A}}[\unrhd \lambda] / \mathscr{H}_{\mathcal{A}}[\triangleright \lambda]\right) \\
& =\operatorname{Ann}_{\mathscr{H}_{\mathcal{A}}} M_{\mathcal{A}}^{\lambda}=\mathscr{H}_{\mathcal{A}}\left[\not \lambda^{\prime}\right]^{\sharp} .
\end{aligned}
$$

Proof. Recall that $M_{\mathcal{A}}^{\lambda}$ has a basis given by all $x_{\lambda} T_{d(t)}$ as $t$ ranges over the set of row-standard tableaux of shape $\lambda$. Now, any $a \in \mathscr{H}_{\mathcal{A}}$ acting as zero on $x_{\lambda} T_{d(t)}$ also acts as zero on $T_{d(s)}^{*} x_{\lambda} T_{d(t)}$, for any $s$. Thus, if $a \in \operatorname{Ann}_{\mathscr{H}_{\mathcal{A}}} M_{\mathcal{A}}^{\lambda}$, then $a$ acts as zero on all $x_{s t}=T_{d(s)}^{*} x_{\lambda} T_{d(t)}$, with $s, t \in$ $\operatorname{Tab}(\lambda)$. Since the images of these elements in $\mathscr{H}_{\mathcal{A}}[\unrhd \lambda] / \mathscr{H}_{\mathcal{A}}[\triangleright \lambda]$ form a

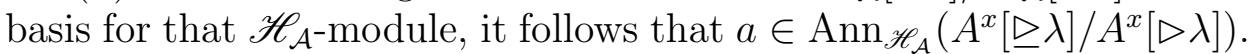
This proves the inclusion $\operatorname{Ann}_{\mathscr{H}_{\mathcal{A}}} M_{\mathcal{A}}^{\lambda} \subseteq \operatorname{Ann}_{\mathscr{H}_{\mathcal{A}}}\left(\mathscr{H}_{\mathcal{A}}[\unrhd \lambda] / \mathscr{H}_{\mathcal{A}}[\triangleright \lambda]\right)$. The opposite inclusion is clear, as $M_{\mathcal{A}}^{\lambda}$ is isomorphic with a submodule of $\mathscr{H}_{\mathcal{A}}[\unrhd \lambda] / \mathscr{H}_{\mathcal{A}}[\triangleright \lambda]$. This proves the second equality.

By Lemma [7.1, we know that $\operatorname{Ann}_{\mathscr{H}_{\mathcal{A}}} M_{\mathcal{A}}^{\lambda} \subseteq \operatorname{Ann}_{\mathscr{H}_{\mathcal{A}}} M_{\mathcal{A}}^{\mu}$ for any $\lambda \unlhd \mu$. Thus, by the result of the previous paragraph, it follows that any $a \in \operatorname{Ann}_{\mathscr{H}_{A}} M_{\mathcal{A}}^{\lambda}$ acts as zero on all $x_{u w}$ for $(u, w)$ of shape $\mu$, for any $\mu \unrhd \lambda$. Since $\mathscr{H}_{\mathcal{A}}[\unrhd \lambda]$ is generated over $\mathcal{A}$ by such $x_{u w}$, it follows that $\operatorname{Ann}_{\mathscr{H}_{\mathcal{A}}} M_{\mathcal{A}}^{\lambda} \subseteq \operatorname{Ann}_{\mathscr{H}_{\mathcal{A}}} \mathscr{H}_{\mathcal{A}}[\unrhd \lambda]$. The opposite inclusion is clear, so this proves the first equality. The third equality is from Theorem 7.3 . 


\section{REFERENCES}

[1] R. Dipper and G. James, Representations of Hecke algebras of general linear groups, Proc. London Math. Soc. (3) 52 (1986), 20-52.

[2] R. Dipper and G. James, The $q$-Schur algebra, Proc. London Math. Soc. (3) 59 (1989), 23-50.

[3] R. Dipper and G. James, $q$-tensor space and $q$-Weyl modules, Trans. Amer. Math. Soc. (1) 327 (1991), 251-282.

[4] R. Dipper and G. James, Blocks and idempotents of Hecke algebras of general linear groups, Proc. London Math. Soc. (3) 54 (1987), 57-82.

[5] A. Giaquinto, Quantization of tensor representations and deformation of matrix bialgebras, J. Pure Appl. Algebra, 79 (1992), 169-190.

[6] J.J. Graham and G.I. Lehrer, Cellular algebras, Invent. Math. 123 (1996), 1-34.

[7] I. Grojnowski and G. Lusztig, On bases of irreducible representations of quantum $G L_{n}$, in "Kazhdan-Lusztig theory and related topics", Contemp. Math. 139 (1992), 167-174.

[8] M. Härterich, Murphy bases of generalized Temperley-Lieb algebras, Archiv Math. 72 (1999), 337-345.

[9] G.D. James, The Representation Theory of the Symmetric Groups, Lecture Notes in Math. 682, Springer-Verlag, Berlin 1978.

[10] M. Jimbo, A $q$-analogue of $U(\mathfrak{g l}(N+1))$, Hecke algebra, and the Yang-Baxter equation. Lett. Math. Phys. 11 (1986), 247-252.

[11] A. Mathas, Iwahori-Hecke algebras and Schur algebras of the symmetric group, University Lecture Series, 15, American Mathematical Society, Providence, RI, 1999.

[12] G.E. Murphy, On the representation theory of the symmetric groups and associated Hecke algebras, J. Algebra 152 (1992), 492-513.

[13] G.E. Murphy, The representations of Hecke algebras of type $A_{n}, J$. Algebra 173 (1995), 97-121.

[14] N. Xi, Root vectors in quantum groups, Comment. Math. Helv. 69 (1994), 612-639.

Mathematics and Statistics, Loyola University Chicago, Chicago, ILLINOIS 60626 U.S.A.

E-mail address: sdoty@luc.edu

Mathematics Department, Willamette University, 900 State Street, Salem, Oregon 97301 U.S.A.

E-mail address: knyman@willamette.edu 